\title{
Automation of $\mu$-SPE (Smart-SPE) and Liquid-Liquid Extraction Applied for the Analysis of Chemical Warfare Agents
}

\author{
Marc André Althoff ${ }^{1}$, Andreas Bertsch ${ }^{2}$ and Manfred Metzulat ${ }^{2, *}$ \\ 1 Department of Chemistry, Ludwig-Maximilian University (LMU), Butenandtstrasse 5-13 (Haus D), \\ D-81377 Munich, Germany; Marc.Althoff@cup.uni-muenchen.de \\ 2 Chemistry Section, Science Department, Chemical Defense, Safety and Environmental Protection School, \\ Mühlenweg 12, D-87527 Sonthofen, Germany; andreasbertsch@bundeswehr.org \\ * Correspondence: manfredmetzulat@bundeswehr.org
}

Received: 30 June 2019; Accepted: 30 August 2019; Published: 9 October 2019

\begin{abstract}
Existing autosamplers are frequently applied only for subjecting the samples to the instruments for injection. In our study, we have set up a TriPlusRSH autosampler mounted on a GC-FID-MS/MS system using the new Method Composer and Script Editor software to automatize all necessary sample preparation steps and subsequent injection of samples in the field of chemical disarmament. Those include but are not limited to: liquid-liquid extraction, drying steps, solvent exchange, and $\mu$-SPE. Tedious and error prone off-line steps are eliminated. In particular, when investigating highly toxic substances like chemical warfare agents or anticancer drugs, automation can help to minimize health risks for lab personnel. The setup engaged features brand new prototype equipment, e.g., a centrifuge to assist in phase separation for liquid-liquid extraction. Efficiency and accuracy of the automated methods were carefully evaluated and proven to outperform the respective manual steps after optimization, e.g., the processing time is up to $60 \%$ faster and recovery rates are doubled. The developed workflows can easily be adapted to other sample preparation protocols, e.g., determination of octanol/water partition coefficients, and be used amongst different instruments and chromatography data handling systems.
\end{abstract}

Keywords: automation; sample handling; sample preparation; chemical warfare agent; SPE; LLE

\section{Introduction}

Preparing and processing of samples prior to analysis is one of the most crucial steps in analytical chemistry and at the same time is the most time consuming task [1]. Most often it is also one of the most cost-effective parts if it has to be done manually. Thus, many people are of the opinion that automation means replacing people by machines [2]. Depending on the analytes being processed automation is a desired feature to have, especially to not expose humans to the risks inhering contagious samples [3] or deadly poisonous compounds such as chemical warfare agents. The latter ones are in our main focus. Lots of methods have been developed for the general work-up of chemical warfare agent samples, their precursors, and degradation products, and are summarized in the book by Vanninen [4]. However, automation has not yet entered the field of chemical warfare agent analysis besides the use of autosamplers for the injection of samples [4]. This may be related to the fact that the number of samples in routine analysis is very low, because luckily chemical warfare agents are very rarely used. Additionally, the implementation of automation is thought to be costly and time-consuming by customers.

In our study we want to show that automation has more advantages than just saving time in laboratory processes. Furthermore, available $x-y-z$ autosamplers [5] can be used instead of separate 
stand-alone instruments, like those available for solid phase extraction (SPE) from, e.g., Gilson or Agilent, or liquid-liquid extraction (LLE) from, e.g., Aurora, which are not capable of sample analysis. However, only a few studies in this research area are both publicly available and report on efficiency of the methods [6]. Häkkinen was the first to publish research on the SPE method development for different chemical warfare agents [7]. Bae et al. report on SPE of VX ([2-(Diisopropylamino)ethyl]-O-ethyl methylphosphonothioate) from various food matrices by HPLC-MS (High Performance Liquid Chromatography - Mass Spectrometry) [8]. A most recent study of the extraction from non-polar solvents was prepared by Sinha et al. [9] and Liu et al. [10] studied the recovery of sulfur mustard metabolites from urine. However, none of these studies report on the extraction of different chemical warfare agents from natural waters or compare different extraction methods with each other. This may be because of the fact that a binary yes-or-no answer is in most cases sufficient regardless of whether a chemical warfare agent was deployed or not, as the amount used is not crucial for its deadly task; c.f. reports of the OPCW (Organization for the Prohibition of Chemical Weapons) to the UNO (United Nations Organization) on the latest investigations in Syria [11].

\section{Materials and Methods}

\subsection{Materials and Analytes}

Reagents used were of purum grade and solvents of analytical grade. They were used without further purification and were purchased from Sigma Aldrich, Schnelldorf, Germany and VWR, Darmstadt, Germany. Chemical warfare agents VX ([2-(Diisopropylamino)ethyl]-O-ethyl methylphosphonothioate), VX-Disulfide (Bis(diisopropylaminoethyl)disulfide), HD (sulfur mustard or Bis(2-chloroethyl) sulfide), and VG (Amiton or O,O-diethyl S-[2-(diethylamino)ethyl] phosphorothioate) were either available from stock from Chemical Defense, Safety and Environmental Protection School Sonthofen, Germany or freshly synthesized by standard in-house methods [12]. Diesel fuel was obtained from a local gas station, soil and 'natural water' were collected from an area outside the laboratory building and from a puddle, respectively. All experiments were run from the same batch of 'natural water'. The specifications of the tap water were provided by the local waterworks [13].

$\mu$-SPE cartridges (C18-EC and Silica) from ITSP Solutions were provided by CTC, Zwingen, Switzerland. A detailed description of the $\mu$-SPE-features can be found on the webpage of ITSP Solutions Inc., Hartwell, GA, USA [14].

\subsection{Hardware Equipment}

Experiments were performed either manually with standard laboratory glassware or on a Thermo Fisher Scientific ${ }^{\circledR}$ GC-MS/MS instrument equipped with a TriPlusRSH autosampler partly bearing prototype equipment, e.g., a centrifuge. The respective setups for the LLE- and $\mu$-SPE experiments are shown in Figure 1. Additional equipment used were billimex ${ }^{\circledR} \mathrm{LD} / \mathrm{HD}$ vials from LABC, Hennef, Germany in course of the LLE-experiments. These vials have a special design to be precisely compatible with the autosampler and were originally invented for performing dispersive liquid-liquid-microextraction on the CTC autosamplers. LD and HD stand for low density and high density of the extraction solvent, respectively. 


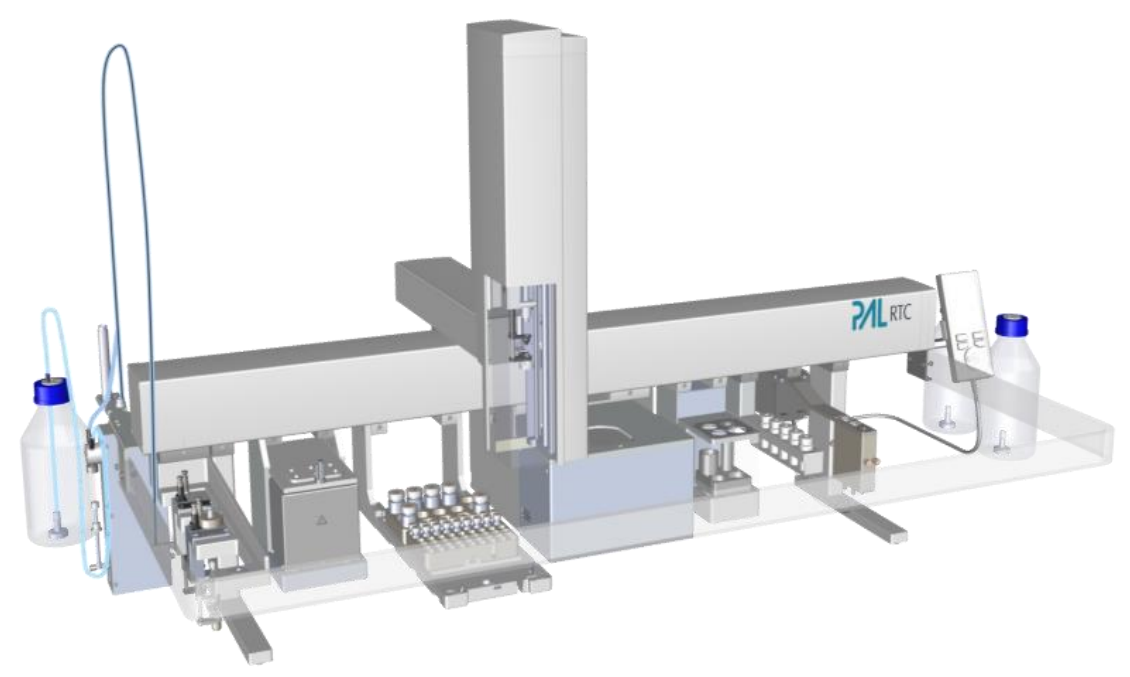

(a)

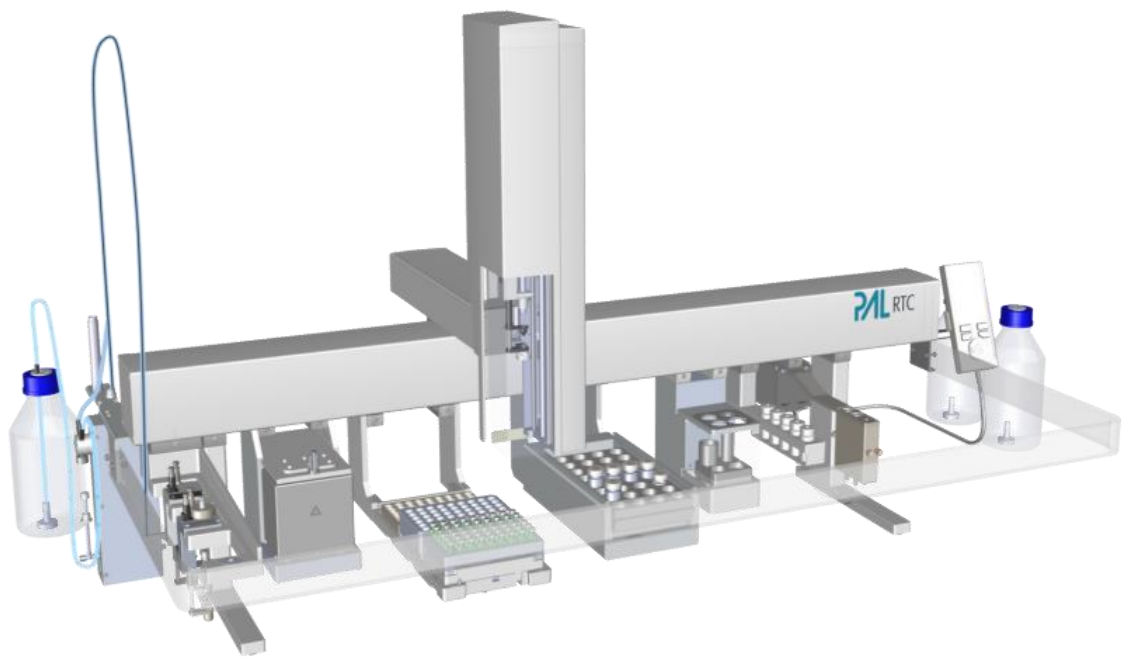

(b)

Figure 1. (a) Autosampler setup for LLE procedures: ATC with Dilutor, Agitator, Sample Rack, Centrifuge, Vortexer, Standard Wash Station and Fast Wash Station (left to right); (b) Autosampler setup for $\mu$-SPE procedures: ATC with Dilutor, Agitator, $\mu$-SPE Rack, Tray-Cooler, Vortexer, Standard Wash Station and Fast Wash Station (left to right).

To set-up the autosampler prototype software, applications from CTC were used. First, the experimental workflows were tested step by step by the PAL Method Composer, version 1.1.17284.927 [15]. This program allows us to drag and drop individual incremental steps of the autosampler processes to a complete workflow without the need to involve a chromatographic data software (CDS). It was recently put on the market as "TriPlus RSH Sampling Workflow Editor Software". A demo-video of this software can be found on the homepage of Thermo Fisher Scientifc [16]. Second, the PAL Script Editor Alpha, version 2.4 Alpha, was used to transfer the PAL Method Composer workflow into a compiled script of optimized processing time and compatibility with the CDS: Chromeleon, version 7.2, SR 4, from Thermo Fisher Scientific. The PAL Method Compser software uses a programming syntax comparable to the well-known $C$ programming language. Scripts were tested offline with the PAL Script Executer, version 2.5 Beta, beforehand. All Scripts are available from the corresponding author upon request. 
Instrument parameters for the analysis of the samples are given in Table 1.

Table 1. Instrument parameters for processing of the samples on GC-FID-MS/MS.

\begin{tabular}{|c|c|}
\hline Instrument Parameter & Value \\
\hline GC & Thermo Fisher Scientific Trace 1310 with PTV Injector \\
\hline Data System & Chromeleon 7.2 SR 4 \\
\hline $\begin{array}{l}\text { Analytical column } \\
\text { Oven conditions: }\end{array}$ & Agilent J\&W GC-column (CP-Sil 8 CB Low Bleed/MS, 30 m $0.25 \mu \mathrm{m}$ ) \\
\hline Temperature program & $70(1)-290{ }^{\circ} \mathrm{C}$ at $30^{\circ} \mathrm{C} \min ^{-1}$, post temperature $290{ }^{\circ} \mathrm{C}(1)$ \\
\hline Carrier gas & Helium BIP ${ }^{\circledR}[17]$ \\
\hline \multicolumn{2}{|l|}{ Injection conditions: } \\
\hline Injector temperature & $250{ }^{\circ} \mathrm{C}$ \\
\hline Injection type & liquid injection: splitless, injection volume $1 \mu \mathrm{L}$ \\
\hline Purge flow & $1.20 \mathrm{~mL} / \mathrm{min}$ \\
\hline Liner type & $130 \mathrm{~mm} \times 2.0 \mathrm{~mm}$ ID glass liner \\
\hline MS & $\begin{array}{l}\text { Thermo Fisher Scientific TSQ Duo, Triple Quadrupole } \\
\text { scan time: } 0.2 \mathrm{~s}\end{array}$ \\
\hline Transfer line temperature & $280^{\circ} \mathrm{C}$ \\
\hline Ion source temperature & $280^{\circ} \mathrm{C}$ \\
\hline Ionization mode & EI \\
\hline FID & $\begin{array}{l}\text { Thermo Fisher Scientific FID-Module } \\
\text { temperature: } 250{ }^{\circ} \mathrm{C} \\
\mathrm{H}_{2} \text {-flow: } 35 \mathrm{~mL} \\
\mathrm{~N}_{2} \text {-flow: } 40 \mathrm{~mL} \\
\text { synthetic air-flow: } 350 \mathrm{~mL}\end{array}$ \\
\hline
\end{tabular}

\subsection{Experimental Workflows}

Experimental workflows follow recommended operating procedures according to the respective chapters of the 'blue book' [4], which are given in brief in the Appendix in a graphical depiction. Necessary adjustments of these procedures, e.g., in volume, are given in the results section, as those had to be adjusted to the equipment used. All experiments were run at least in triplicate to yield a reliable RSD.

- Liquid-liquid Extraction:

Tests of the extraction efficiency in the automated liquid-liquid extraction were performed in the range of 600-2000 rpm of the autosampler vortexer. A mixture of caffeine and theophylline in the ratio of $4: 1$ was used as the evaluation test mixture to find the optimum vortex speed. The mean of three replicates per speed value were determined.

Preparation of samples: stock solutions of tap water and 'natural water' were spiked with the respective analytes stock solution to reach a $10 \mathrm{ppm}$ level and adjusted to $\mathrm{pH} 7$ and $\mathrm{pH} 11$, respectively by the addition of ammonium hydroxide solution, each as a requirement of the recommended procedures (c.f. Figures A1-A3). For preparing the soil samples the respective amount of analytes were directly spiked to the stock sample of soil. The wipe sample was a cotton bud tip onto which a 10 ppm solution of the analytes was directly loaded with a gas-tight Hamilton syringe. The analytes stock solution was prepared in dichloromethane.

- $\mu$-SPE:

Generally the workup procedures of the 'blue book' [4] could be used to extract the analytes with the respective $\mu$-SPE cartridges. However, adjustments and optimizations of flow rates and volumes had to be found. The flow rates for loading, elution and washing steps were optimized in the range of two to $20 \mu \mathrm{L} / \mathrm{s}$. Therefor the recovery rates in terms of the peak area of the given analytes was determined. Samples prepared in tap water were processed with C18-EC $\mu$-SPE cartridges and those dissolved in $n$-hexane were spiked with a strong hydrocarbon background of $10 \mathrm{mg} / \mathrm{mL}(1 \%$ o) 
of diesel fuel and were processed with silica $\mu$-SPE cartridges. Samples were spiked with $10 \mathrm{ppm}$ of the respective analytes. To remove the hydrocarbon background by means of the silica $\mu$-SPE cartridge, the following procedure was used: (i) the silica cartridge was preconditioned with $1 \mathrm{~mL}$ of $n$-hexane, (ii) $500 \mu \mathrm{L}$ of sample were loaded on the cartridge, (iii) the cartridge was washed with $2 \mathrm{~mL}$ of $n$-hexane and (iv) the cartridge was eluted with $500 \mu \mathrm{L}$ of methanol. Additional parameters, which were developed and optimized in course of this study, are given in the results section.

\section{Results and Discussion}

Generally, it can be said that the transfer and automation of the described methods on the first glance seems to be very easy. However, the transfer of a basic laboratory procedure, e.g., liquid-liquid extraction, has to be broken down to the very incremental steps. Therefore, a very careful examination of the incremental work steps is necessary.

\subsection{Automated Liquid-Liquid Extraction}

The given protocol for manual liquid-liquid extraction according to reference [4] was adapted to the autosampler setup with respect to the volume of the vial size suitable for the autosampler $(2-20 \mathrm{~mL})$. For complexity reasons the respective liquid-liquid extraction workflow for the PAL Method Composer software is only given in its very basic structure, c.f. Figure 2. For each item in the list parameters like penetration depth of the vial, penetration speed of the needle or vortex speed and duration had to be defined and optimized once. In case multiple extractions have to be prepared, the routine can be altered by adding a repeat command for entries "Dilutor Add Solvent" to the second "Clean Syringe" command. The easy to use PAL Method Composer software was very convenient in the testing of the identified incremental work steps. A brief description of the workflow is as follows: The autosampler picks up the dilutor tool to prime it (rinsing), before it adds a given amount of extraction solvent to the sample vial. Afterwards the vial is vortexed and optionally centrifuged, meaning one can decide whether centrifugation is necessary or not. In the next step the tool is changed to a regular syringe to transfer the extraction solvent layer to a second vial containing a proper amount of sodium sulfate (drying agent). After a final vortexing step, the sample is injected into the GC. Finally, the autosampler is returning to home position. The entry liquid injection contains another tool change to get the correct syringe tool with the injection syringe and not the extraction solvent transfer syringe.

For comparison with the manually performed liquid-liquid extractions the workflow was transferred into a regular script for the autosampler by PAL Script Editor software, which is fully operational with the Chromeleon CDS. First, the general extraction performance of the vortexer tool was evaluated by variation of the vortex speed and vortexing time. As can be seen from Figure 3 the mechanical limits of the vortexer are 600 to $2000 \mathrm{rpm}$. The extraction efficiency for five seconds extraction time is lower compared to ten seconds extraction time. However, at about $1200 \mathrm{rpm}$ the differences can be neglected and the optimum extraction conditions are reached. Thus, for all further experiments these conditions were used. The manual procedure recommends a two-fold extraction of five minutes, meaning the automated workflow is up to 60 times faster, having the same extraction efficiency. The described workflow can easily be extended by other sample preparation tasks, e.g., derivatization steps, as we have described previously [18]. 


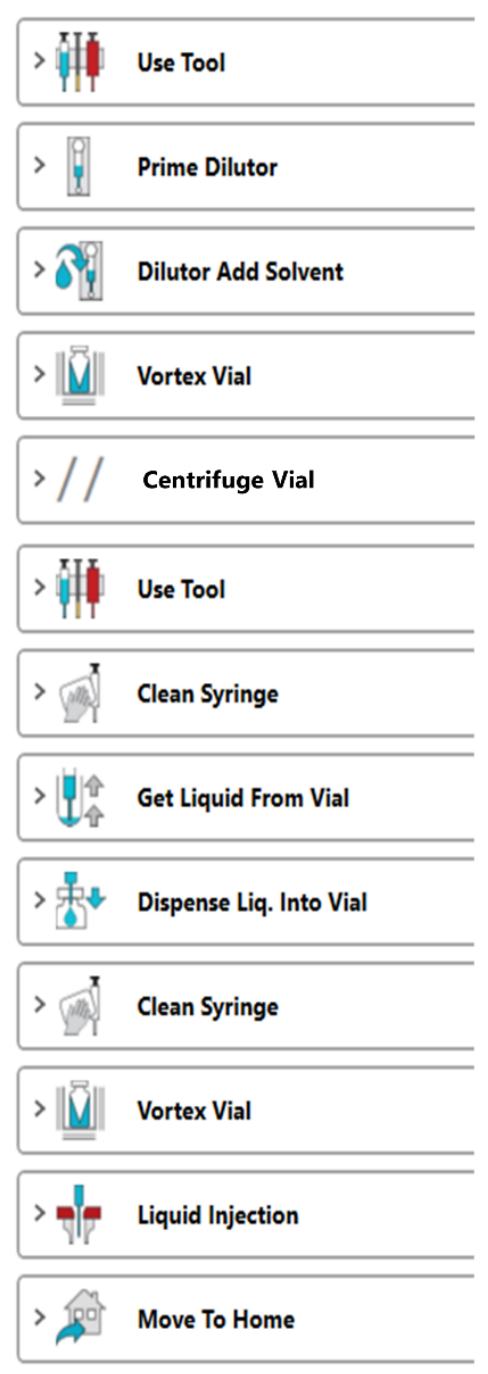

Figure 2. Scheme of the basic steps for the liquid-liquid extraction experiment represented in the PAL Method Composer software style. Each menu item consists of a set of parameters which have to be adjusted and optimized.

Consequently, we applied the developed automated workflow for the simultaneous extraction of different type of chemical warfare agents VX, VG and HD from four different matrices. To the best of our knowledge, this has not been done before. The results found (c.f. Table 2) clearly show that very high recovery rates of the analytes in the given matrix are achieved. Recovery rates vary depending on the $\mathrm{pH}$-value of the matrix. The more acidic the matrix the faster the degradation process of the V-series nerve agents is and, thus, only less amount of analyte can be recovered. These findings are in very good agreement with our previous study on solid-phase microextraction of the same analytes [18]. In contrast, HD hydrolyses faster at higher $\mathrm{pH}$-values. For soil and wipe samples strong matrix effects have to be taken into account and, thus, a fair amount of analytes is lost to the matrix and cannot be recovered. Overall the obtained recovery rates are excellent with respect to processing time and automation of the overall process. 


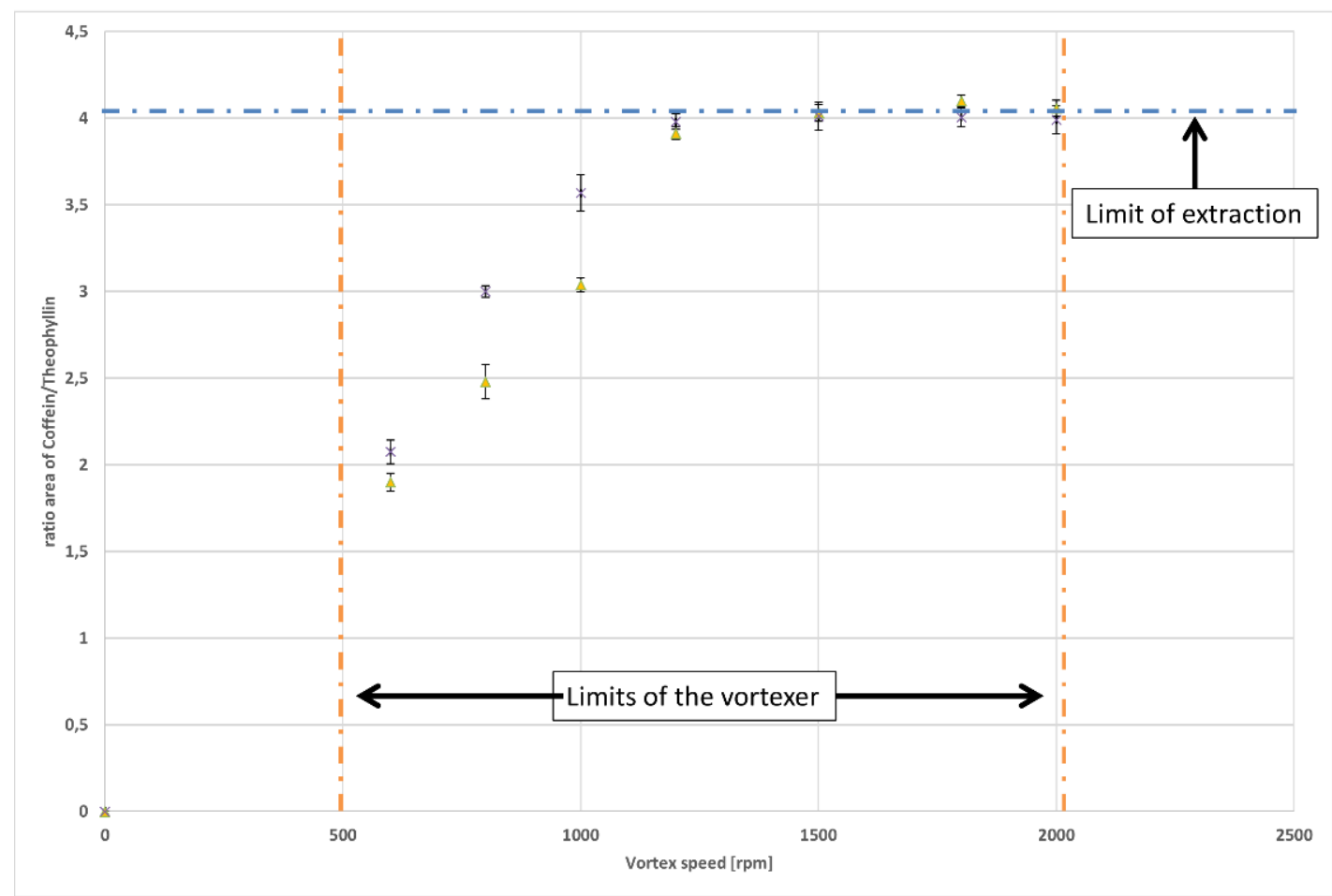

Figure 3. Results of the automated liquid-liquid extraction methodology. Noteworthy are the short extraction times of 5 to $10 \mathrm{~s}$ and completeness of extraction at $1200 \mathrm{rpm}$.

Table 2. Results of the automated extraction of chemical warfare agents VX, VG and HD at 5 s extraction time and $1200 \mathrm{rpm}$ vortex speed.

\begin{tabular}{cccc}
\hline Matrix ${ }^{\mathbf{1}}$ & \multicolumn{3}{c}{ Recovery Rate \pm RSD [\%] } \\
\hline & VX & VG & HD \\
tap water (pH 7) & $64.1 \pm 9.6$ & $79.8 \pm 8.3$ & $47.3 \pm 3.8$ \\
tap water (pH 11) & $88.0 \pm 3.4$ & $85.9 \pm 5.1$ & $22.1 \pm 2.7$ \\
surface water (pH 7) & $96.0 \pm 3.4$ & $99.9 \pm 3.6$ & $51.5 \pm 4.0$ \\
surface water (pH 11) & $98.7 \pm 1.4$ & $99.9 \pm 1.8$ & $50.7 \pm 1.4$ \\
soil & $66.2 \pm 2.7$ & $88.2 \pm 4.0$ & $46.8 \pm 4.4$ \\
wipe & $65.6 \pm 3.1$ & $58.4 \pm 2.3$ & $51.2 \pm 1.4$ \\
\hline
\end{tabular}

${ }^{1}$ Aqueous samples were adjusted to the given $\mathrm{pH}$-value prior to the extraction.

Another advantage of the automation is that comparably small amounts of sample can be handled. With our setup, we were able to easily process quantities down to $1 \mathrm{~mL}$. Thus we meet all of the three key tasks of today's laboratory issues: automation, miniaturization and processing time as were described by Costa and Pawliszyn $[19,20]$. Best results could be achieved by using billimex ${ }^{\circledR}$ LD/HD vials, which allow an almost complete "decantation" of lower or upper phase of the extraction solvent by means of the autosampler syringe. They are fully compatible with standard autosampler equipment. A picture of the different vial types used during this study and their appearance during the extraction process is shown in Figure 4. Generally, to "decant by syringe" the exact penetration depth needed to be determined to exactly hit the interface layer of the two liquids. We are of the opinion that the miniaturization and optimization of well-established sample preparation protocols is superior to the development of new extraction methods like Liquid-Phase Microextraction or Single-Drop Microextraction, since even more and yet not existing hardware modules would be needed to be invented. Furthermore, those tools would have to be very complex, e.g., to hit a single drop on the surface of a liquid with a syringe is very hard to do manually; therefore a camera and a vial which is movable and tilt-able with respect to the syringe position would include at least a second robot 
arm which is more flexible than existing ones. This would make the instrumental setup more cost intensive and would also cost a lot of time in processing a sample. To compensate this shortcoming of the employed $x-y-z$-autosampler, freely movable industry-robot-arms, like in automobile industry could be used, but are even more complex and are not yet commercially available of the shelf for chemical analysis.
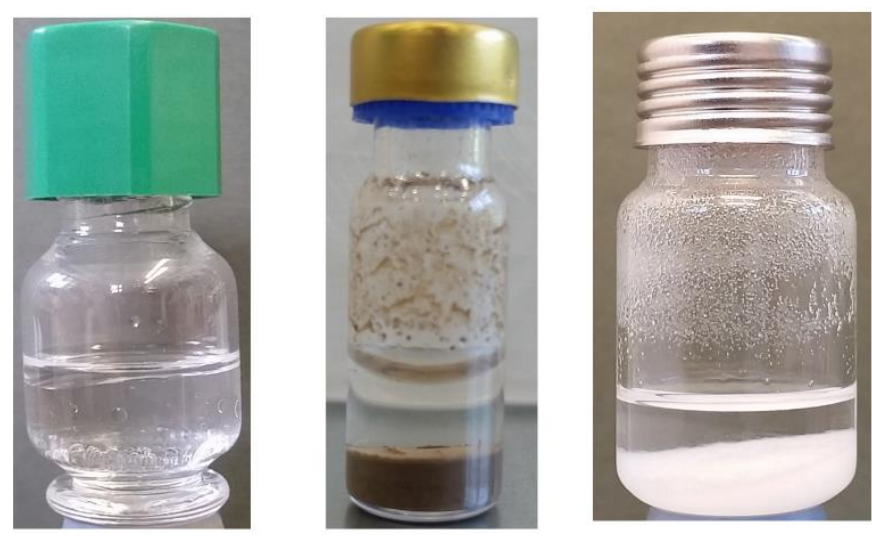

Figure 4. Autosampler vials after (i) extraction (left, billimex ${ }^{\circledR}$ ), (ii) centrifugation (middle) and (iii) drying (vortexed) with $\mathrm{Na} 2 \mathrm{SO} 4$ (right).

Another challenging part was to obtain a good phase separation after vortexing, especially in case of processing the soil samples. However, the prototype centrifuge we had access to during our studies proved to be the right tool to help phase separation and to force all floating particles to the bottom of the extraction vial, c.f. Figure 4 (middle). Additionally, also other common non-desirable effects, which can occur during liquid-liquid extraction, e.g., foaming, can be dealt with by using the centrifuge. Due to being prototype equipment the centrifuge commands were not available in the PAL Method Composer software. Thus, it had to be programmed using the PAL Script editor. Centrifuge parameters were not optimized during this study since they would have not affected the recovery rates. The centrifuge was not used after the extraction solvent was added to the drying agent since sodium sulfate very nicely precipitated during transportation of the vial from the vortexer to the original vial position. One possible limitation of the autosampler centrifuge could be its G-force limit of $2000 \mathrm{G}$ with respect to the use of larger sampling vials $(10$ and $20 \mathrm{~mL})$. According to the blue book [4] up to $5000 \mathrm{G}$ should be applied for the phase separation process. This is only possible to achieve by using $2 \mathrm{~mL}$ autosampler vials.

\subsection{Automated $\mu-S P E$}

The basic workflow of the $\mu$-SPE experiment for removing the hydrocarbon background is described in the experimental part, whereas the procedure for the C18 method is given in Figure A3 of Appendix A. The main difference between standard SPE and $\mu$-SPE is the cartridge size and sorbent volume. The $\mu$-SPE cartridge contains only $10-45 \mathrm{mg}$ of sorbent material. Flow rates are precisely controlled by the autosampler plunger motor; as low as $1 \mu \mathrm{L} / \mathrm{s}$. This high precision helps to achieve high accuracy of the overall process. The four incremental steps of the experiment are: conditioning of the cartridge, loading of the sample onto the cartridge, washing off undesired compounds and elution of the analytes. The C18-method had to be adjusted regarding the applied volumes: (i) conditioning: $1 \mathrm{~mL}$ of methanol and $2 \mathrm{~mL}$ of water, (ii) loading: $500 \mu \mathrm{L}$ of sample, (iii) elution: $1 \mathrm{~mL}$ of ethyl acetate. Drying of the cartridge was performed by pushing $5 \mathrm{~mL}$ of air through the cartridge with the autosampler syringe.

In the beginning the flow rates had to be optimized. An exemplary plot of the results for the elution flow rates is shown in Figure 5. As can be seen the elution flow rate is optimum at $12 \mu \mathrm{L} / \mathrm{s}$ for all analytes. The different heights of the curves for VX and VG are a matter of the response factor of the 
detector for the individual analyte, whereas VX-disulfide, as a degradation product of VX, was present at $1 / 10$ of the concentration of VX. Moreover, we also found that for loading of the cartridge, a flow rate of $12 \mu \mathrm{L} / \mathrm{s}$ was best.

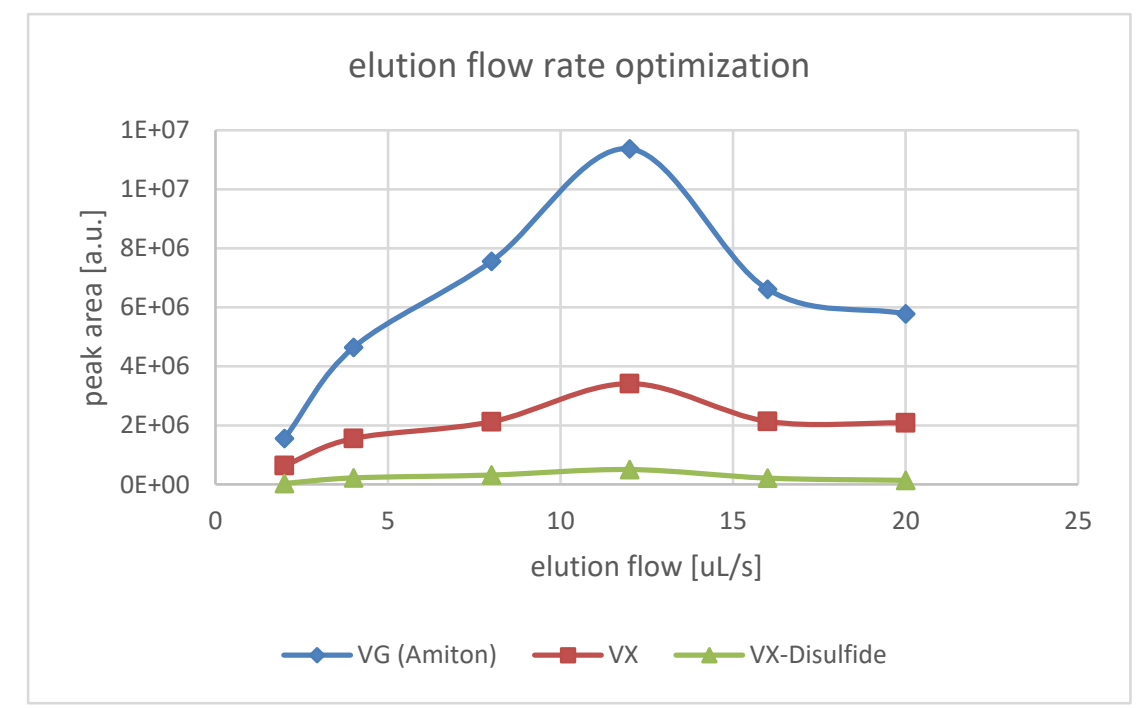

Figure 5. Representation of the elution flow rates and obtained peak areas in GC-MS for the different analytes on the silica $\mu$-SPE cartridge.

In Figure 6 three different scans from the hydrocarbon contaminated sample show that the chosen sample preparation technique (spiking) is successful, meaning that a direct injection of the organic sample in full-scan mode (top) or SIM mode (middle) is not successful in the detection of any analyte from the sample. The last chromatogram (bottom) was obtained in SIM mode after sample processing with the developed $\mu$-SPE methodology and very nicely shows the "hidden" analytes of the original sample in a very good response.

In Table 3 the results of the two different sample processing procedures are summarized. First, all for analytes were extracted from an aqueous sample buffered to neutral conditions as recommended by extraction with C18-EC cartridge. On average, the recovery rates range from 56 to $65 \%$ of the spiked amount. Compared to available literature resources, this is roughly twice the amount of analyte as can be obtained with the manual procedure. For VX-Disulfide and VG, no literature values have been reported yet. For sample purification in case of a strong hydrocarbon background as impurity even higher recovery rates could be obtained ranging from 76 to $98 \%$ of the originally spiked amount of analyte. Due to the non-polar properties of sulfur mustard (HD), this analyte is not efficiently retained on the silica cartridge. Moreover, it is washed off the silica cartridge together with the hydrocarbon background by the washing solvent ( $n$-hexane). The eluted fraction containing the hydrocarbon background as well as the sulfur mustard has to be treated in another preparation step which can also be found in the blue book. However, here we report recovery rates for the cleaning of a hydrocarbon background from chemical warfare agents by silica cartridges for the first time. 

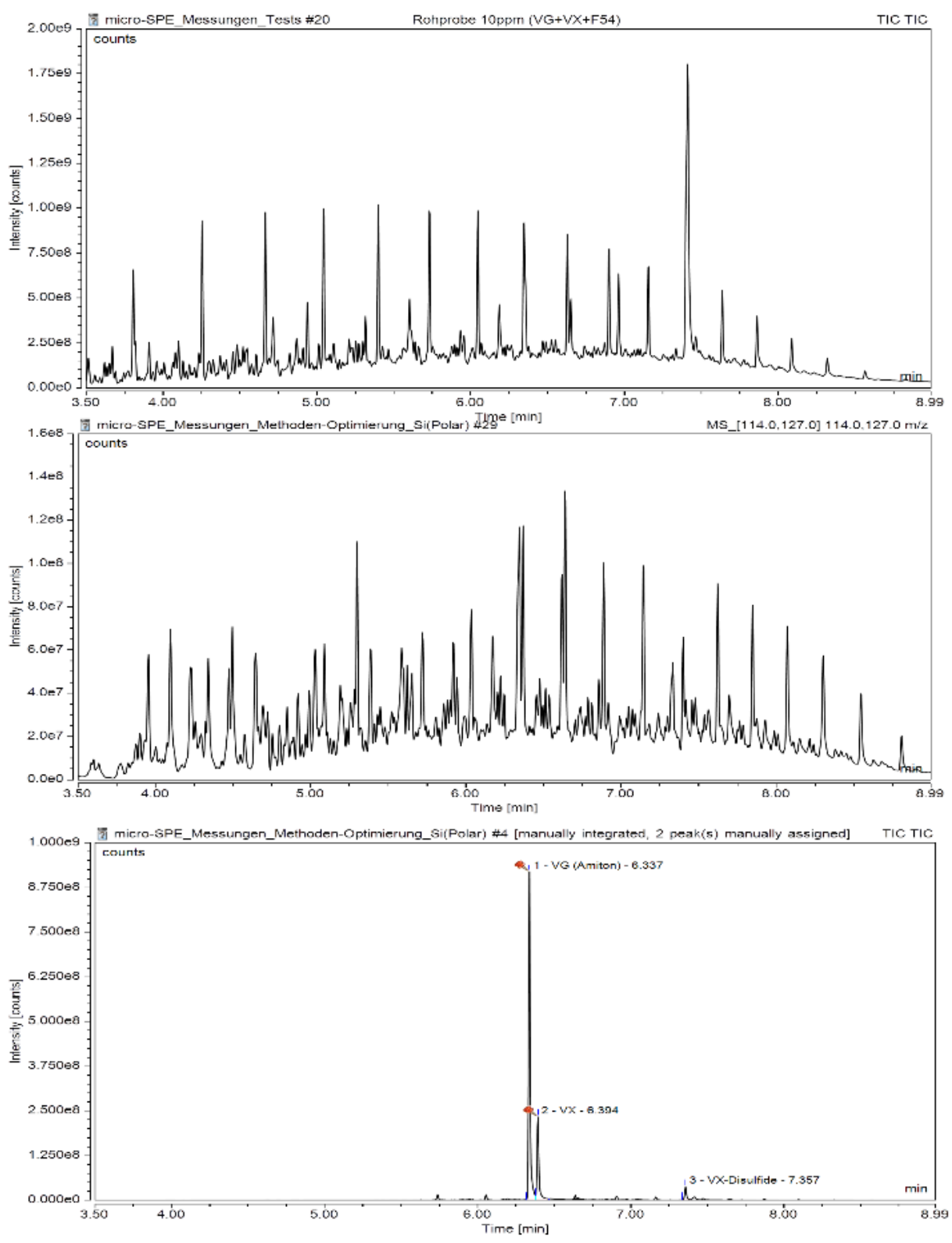

Figure 6. Chromatograms of the sample containing a strong hydrocarbon background from diesel fuel: TIC-scan of the raw sample (top), SIM-scan of the raw sample (middle) and SIM-scan after sample preparation with silica cartridge (bottom).

Table 3. Recovery rates of chemical warfare agents from aqueous samples and strong hydrocarbon background containing organic solvent.

\begin{tabular}{ccccc}
\hline Matrix (Cartridge) & \multicolumn{4}{c}{ Recovery \pm RSD [\%] } \\
\hline & VX & VX-Disulfide & VG & HD \\
\hline water (C18-EC) & $56.5 \pm 0.5$ & $57.9 \pm 2.2$ & $65.8 \pm 0.1$ & $64.8 \pm 1.9$ \\
water samples [7] & $24 \pm 10$ & -1 & -1 & $32 \pm 7$ \\
diesel fuel (silica) & $76.3 \pm 1.3$ & $77.4 \pm 0.2$ & $98.2 \pm 1.5$ & -2 \\
\hline
\end{tabular}

${ }^{1}$ no values available, ${ }^{2}$ analyte not retained by the cartridge.

The main technical differences between the well-established recommended operating procedures of the blue book and our experimental setup are given in Table 4. In sense of going green with sample preparation processes, our methodology is advantageous over existing methods: less sorbent material 
and less solvent volume are needed. Comparable autosamplers, e.g., from Gerstel, are (yet) not capable of processing $\mu$-SPE cartridges. Instead they are capable of processing standard-sized SPE-cartridges. This means that in total comparably fewer samples can be processed due to the fact that more room is needed for keeping available fresh cartridges. Moreover, a high enrichment factor of up to 200 can be achieved on a $10 \mathrm{mg}$ cartridge as well as the flow rate can be precisely controlled and has a highly sufficient repeatability. Additionally, the time for the overall experiment is cut down from 15 to $10 \mathrm{~min}$. Furthermore, it seems to be a very convincing fact that most manually handling steps of highly toxic substances can be cut down to a minimum and thus the risk for contamination of laboratory personal is minimized.

Table 4. Comparison of $\mu$-SPE and standard SPE.

\begin{tabular}{cccc}
\hline Parameter & $\begin{array}{c}\mu \text {-SPE } \\
\text { (This Work) }\end{array}$ & $\begin{array}{c}\text { SPE } \\
\text { (Gerstel MPS [21]) }\end{array}$ & SPE (Laboratory) \\
\hline sorbent volume & $10-45 \mathrm{mg}$ & $0.1-2 \mathrm{~g}$ & $0.1 \mathrm{~g}-\mathrm{bulk}$ \\
solvent volume & $125 \mu \mathrm{L}$ & $\leq 10 \mathrm{~mL}$ & $3 \mathrm{~mL}$ \\
processing time & $10 \mathrm{~min}$ & unknown & $15 \mathrm{~min}$ \\
flow rate & $1-100 \mu \mathrm{L} / \mathrm{s}$ & $10-250 \mu \mathrm{L} / \mathrm{s}$ & ca. $1 \mathrm{drop} / \mathrm{s}$ \\
enrichment factor & $\leq 200$ & $5-10$ & Indefinite \\
\hline
\end{tabular}

\section{Conclusions and Future Perspective}

Although many steps of the described procedures could be automated and are working very well, some experimental limitations do remain unresolved - so far. To the best of our knowledge, there is no device available to be mounted on the kind of autosampler we used to measure and adjust the $\mathrm{pH}$ value meaning that one initial step of all the blue book procedures remains manual work. As miniaturization of equipment proceeds, less solvent and auxiliary materials are needed and very reliable methods can be branded as green technologies afterwards.

Another auxiliary step, not explicitly mentioned in the procedures itself but sometimes crucial in sample processing, is filtration. Real-life samples can possibly contain floating components or sediment material, which would disturb the described method by blocking syringes or $\mu$-SPE cartridges. This would be easily noticed during manual processing of the sample but not by automatic procedures. Eventually CTC informed us, that the autosampler can be also adapted with tools from Brechbühler company for processing Thomson filter vials [22], which would easily allow filtering sample fractions of $1 \mathrm{~mL}$ in an automated fashion. For the described method of LLE of $\mu$-SPE, this would mean our script would have to be extended by a few basic operations of the autosampler and this issue could be addressed. However, we were not able to get access to such a setup and could not extend our study in this direction.

The PAL Method Composer software proved to be easily suitable to put complex workflows by means of drag-and-drop together and be tested without the need of blocking the analytical instrument. The PAL Script Editor allowed a complex programming of the instrument based on the preliminary results obtained from the PAL Method Composer software and full integration of the analytical instrument and its CDS. Moreover, the workflows can be transferred with slight alternations to other CDS form different instrument manufacturers, as was done with some other routines, e.g., those described in our previous work [18], in cooperation with one of our partner laboratories of the German Federal Police.

The herein developed workflows are running very stable and have found their way into daily laboratory routines (SOP's) in the processing of chemical warfare agents and other analytes. They are faster, have a higher precision, use less material and chemicals, and pose less risk to the laboratory personnel as manual handling time of chemical warfare agents is absolutely minimized. Moreover, we can imagine that the liquid-liquid extraction procedure in particular can be easily adapted for other experimental tasks, e.g., determination of octanol/water partition coefficients or be employed in the 
analysis of pesticide residues or in the field of anticancer drug research where similar procedures are daily routine.

Finally, we are of the opinion that by our efforts in automation (c.f. [18]) and miniaturization reported in this work have entered a new area in the processing of chemical warfare agents.

Author Contributions: Conceptualization, M.A.A.; Data curation, M.A.A.; Formal analysis, M.A.A.; Investigation, A.B.; Methodology, M.A.A.; Project administration, M.M.; Resources, M.M.; Software, M.A.A. and A.B.; Supervision, M.M.; Validation, M.A.A.; Visualization, A.B.; Writing-original draft, M.A.A.

Funding: This research received no external funding.

Acknowledgments: We acknowledge the collaboration between the German Armed Forces (Bundeswehr) and the Ludwig-Maximilian University (LMU) according to the official collaboration agreement between the two institutions. The authors of this study are indebted to CTC Company, Zwingen, Switzerland for giving access to prototype equipment, providing the $\mu$-SPE cartridges from ITSP ${ }^{\mathrm{TM}}$ solutions Inc. and support in programming and creation of the autosampler sketches. billimex ${ }^{\circledR}$ LD/HD-AS vials were provided by courtesy of LABC-Labortechnik Zillger KG, Hennef, Germany.

Conflicts of Interest: The authors declare no conflict of interest. The companies providing material for this study had no role in the design of the study; in the collection, analyses, or interpretation of data; in the writing of the manuscript, or in the decision to publish the results.

\section{Appendix A}

\section{Sample Preparation}

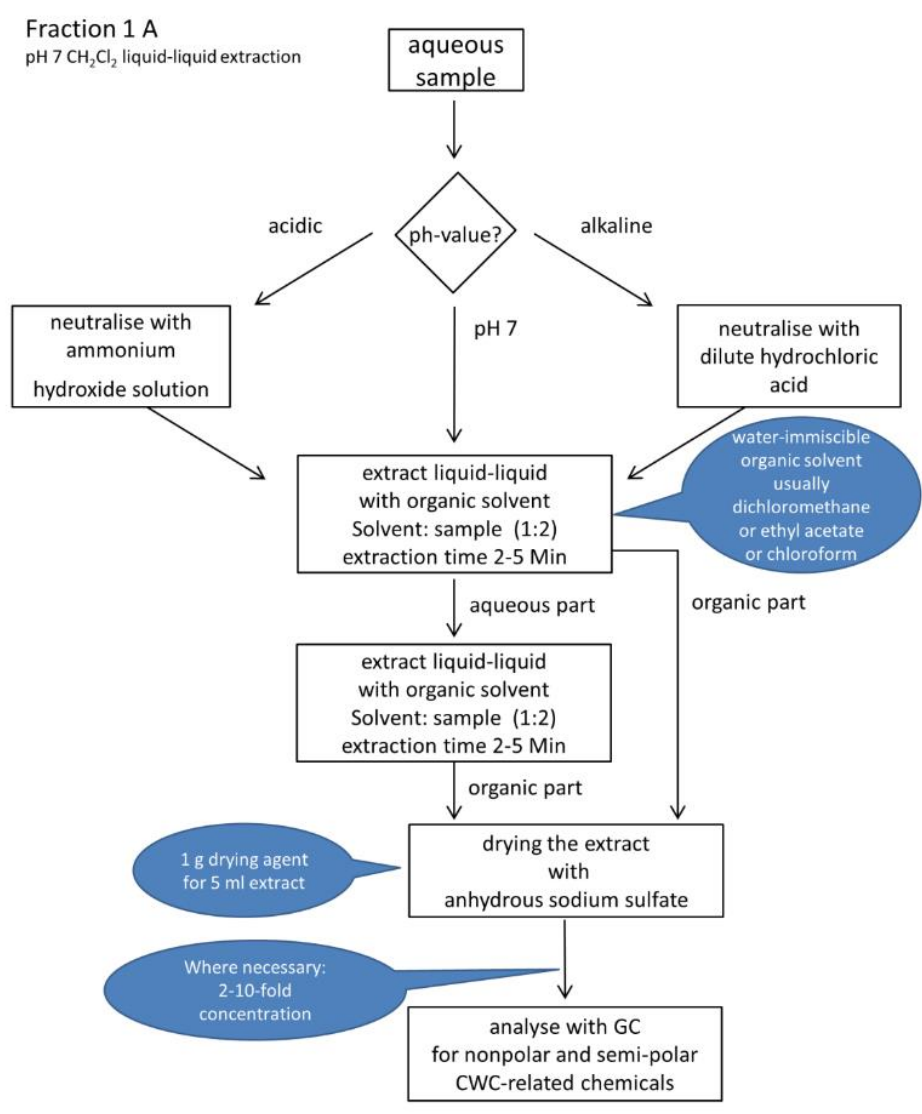

Note! Do not concentrate the organic extract to dryness since certain volatile CWC-related chemicals such as sarin are lost from the sample and, on the other hand, certain less volatile CWC- related chemicals such as VX in residues of organic extracts are firmly adsorbed to glass surfaces.

Figure A1. Recommended operating procedure according to the blue book for the processing of liquid-liquid extraction at neutral conditions. 


\section{Sample Preparation}

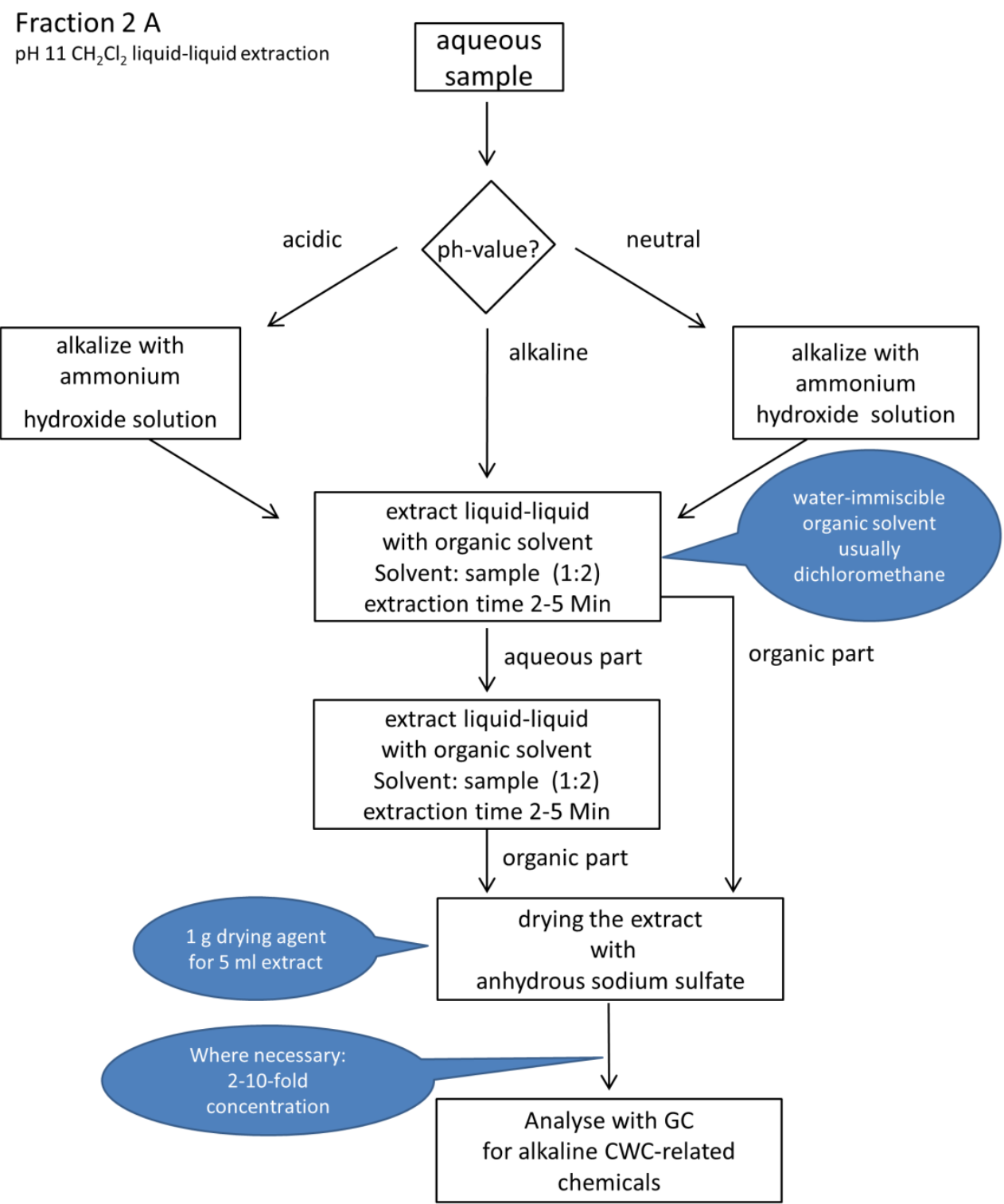

Note! Do not concentrate the organic extract to dryness since certain volatile CWC-related chemicals such as sarin are lost from the sample and, on the other hand, certain less volatile CWC- related chemicals such as VX in residues of organic extracts are firmly adsorbed to glass surfaces.

Figure A2. Recommended operating procedure according to the blue book for the processing of liquid-liquid extraction at alkaline conditions. 


\section{Sample Preparation}

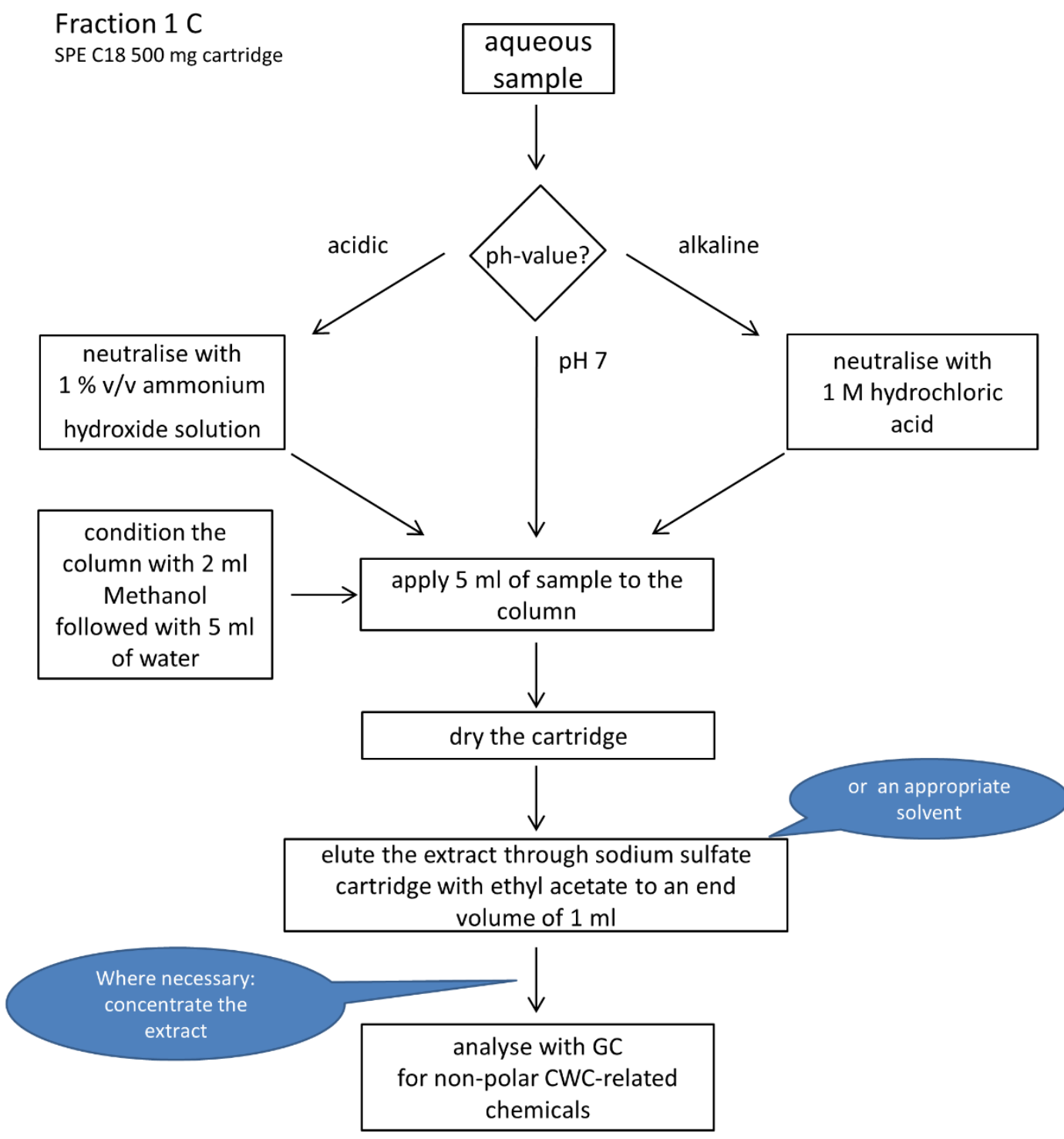

Note! Do not concentrate the organic extract to dryness since certain volatile CWC-related chemicals such as sarin are lost from the sample and, on the other hand, certain less volatile CWC- related chemicals such as VX in residues of organic extracts are firmly adsorbed to glass surfaces.

Figure A3. Recommended operating procedure according to the blue book for the processing of solid-phase extraction by C18-sorbent at neutral conditions.

\section{References}

1. Pawliszyn, J.; Lord, H.L. Handbook of Sample Preparation; Wiley: Hoboken, NJ, USA, 2012.

2. Chui, M.; Manyika, J.; Miremadi, M. Where Machines Could Replace Humans and Where They Can't (Yet)? Available online: http://www.oregon4biz.com/assets/e-lib/Workforce/MachReplaceHumans.pdf (accessed on 25 September 2019). 
3. Armbruster, D.A.; Overcash, D.R.; Reyes, J. Clinical Chemistry Laboratory Automation in the 21st Century-Amat Victoria curam (Victory loves careful preparation). Clin. Biochem. Rev. 2014, 35, 143-153.

4. Vanninen, P. Recommended Operating Procedures for Analysis in the Verification of Chemical Disarmament; University of Helsinki: Helsinki, Finnland, 2017.

5. Wittsiepe, J.; Nestola, M.; Kohne, M.; Zinn, P.; Wilhelm, M. Determination of polychlorinated biphenyls and organochlorine pesticides in small volumes of human blood by high-throughput on-line SPE-LVI-GC-HRMS. J. Chromatogr. B 2014, 945-946, 217-224. [CrossRef] [PubMed]

6. Kuitunen, M.L. Sample Preparation for Analysis of Chemicals Related to the Chemical Weapons Convention in an Off-site Laboratory. In Encyclopedia of Analytical Chemistry; Meyers, R.A., Ed.; Wiley: Hoboken, NJ, USA, 2010. [CrossRef]

7. Häkkinen, V.M.A. Analysis of chemical warfare agents in water by solid phase extraction and two-channel capillary gas chromatography. J. Sep. Sci. 1991, 14, 811-815. [CrossRef]

8. Bae, S.Y.; Winemiller, M.D. Quantification of VX Nerve Agent in Various Food Matrices by Solid-Phase Extraction Ultra-Performance Liquid Chromatography-Time-of-Flight Mass Spectrometry; Edgewood Chemical Biological Center: Edgewood, WA, USA, 2016; p. 26.

9. Sinha Roy, K.; Goud, D.R.; Chandra, B.; Dubey, D.K. Efficient Extraction of Sulfur and Nitrogen Mustards from Nonpolar Matrix and an Investigation on Their Sorption Behavior on Silica. Anal. Chem. 2018, 90, 8295-8299. [CrossRef] [PubMed]

10. Liu, C.C.; Liu, S.L.; Xi, H.L.; Yu, H.L.; Zhou, S.K.; Huang, G.L.; Liang, L.H.; Liu, J.Q. Simultaneous quantification of four metabolites of sulfur mustard in urine samples by ultra-high performance liquid chromatography-tandem mass spectrometry after solid phase extraction. J. Chromatogr. A 2017, 1492, 41-48. [CrossRef] [PubMed]

11. Sellström, A. Report of the United Nations Mission to Investigate Allegations of the Use of Chemical Weapons in the Syrian Arab Republic on the Alleged Use of Chemical Weapons in the Ghouta Area of Damascus on 21 August 2013. Available online: https://www.un.org/zh/focus/northafrica/cwinvestigation.pdf (accessed on 26 September 2019).

12. Althoff, M.A.; Bertsch, A.; Metzulat, M.; Kalthoff, O.; Karaghiosoff, K. New Aspects of the Detection and Analysis of Organo(thio)phosphates related to the Chemical Weapons Convention. Phosphorus Sulfur 2016, 192, 149-156. [CrossRef]

13. Trinkwasseranlayse der Stadt Sonthofen; Stadt Sonthofen: Sonthofen, Germany, 2018; Volume 2018, pp. 1-2.

14. ITSP Solutions Inc. Available online: https://www.itspsolutions.com/ (accessed on 22 May 2019).

15. PAL Method Composer. Available online: https://www.palsystem.com/index.php?id=850 (accessed on 27 July 2019).

16. TriPlus ${ }^{\mathrm{TM}} \mathrm{RSH}^{\mathrm{TM}}$ Sampling Workflow Editor Software. Available online: https://www.thermofisher.com/ order/catalog/product/1R77010-1200 (accessed on 27 July 2019).

17. BIP Gasreinigungstechnologie. Available online: http://www.tig.de/produkte/bip-gasreinigungs-technologie. html (accessed on 25 September 2019).

18. Althoff, M.A.; Bertsch, A.; Metzulat, M.; Klapötke, T.M.; Karaghiosoff, K.L. Application of Headspace and Direct Immersion Solid-Phase Microextraction in the Analysis of Organothiophosphates related to the Chemical Weapons Convention from Water and Complex Matrices. Talanta 2017, 174, 295-300. [CrossRef] [PubMed]

19. Costa, R. Newly Introduced Sample Preparation Techniques: Towards Miniaturization. Crit. Rev. Anal. Chem. 2014, 44, 299-310. [CrossRef] [PubMed]

20. Pawliszyn, J. Handbook of Solid Phase Microextraction; Elsevier: Hoboken, NJ, USA, 2011.

21. Solid Phase Extraction Specifications. Available online: http://www.gerstel.com/pdf/SPE_Spec_en.pdf (accessed on 26 September 2019).

22. Jaffuel, A.; Huteau, A.; Moreau, S. Direct Liquid Chromatography Tandem Mass Spectrometry Analysis of Glyphosate, AMPA, Glufosinate, and MPPA in Water Without Derivatization. Column 2018, 14, 36-40.

(C) 2019 by the authors. Licensee MDPI, Basel, Switzerland. This article is an open access article distributed under the terms and conditions of the Creative Commons Attribution (CC BY) license (http://creativecommons.org/licenses/by/4.0/). 